\title{
Left ventricular strains correlate with aortic elastic properties in adult patients with corrected tetralogy of Fallot (Results from the CSONGRAD Registry and MAGYAR-Path Study)
}

\author{
Gergely Rácz, Árpád Kormányos, Péter Domsik, Anita Kalapos, Nándor Gyenes, Kálmán Havasi, \\ Nóra Ambrus, István Hartyánszky, Gábor Bogáts, Attila Nemes \\ 2nd Department of Medicine and Cardiology Centre, Medical Faculty, Albert Szent-Györgyi Clinical Center, University of Szeged, Szeged, Hungary \\ Contributions: (I) Conception and design: A Nemes; (II) Administrative support: N Ambrus; (III) Provision of study materials or patients: A Nemes, \\ K Havasi; (IV) Collection and assembly of data: G Rácz, Á Kormányos, P Domsik, A Kalapos; (V) Data analysis and interpretation: G Rácz, Á \\ Kormányos, A Nemes; (VI) Manuscript writing: All authors; (VII) Final approval of manuscript: All authors. \\ Correspondence to: Attila Nemes, MD, PhD, DSc, FESC. 2nd Department of Medicine and Cardiology Center, Medical Faculty, Albert Szent-Györgyi \\ Clinical Center, University of Szeged, Semmelweis Street 8, P.O. Box 427, H-6725 Szeged, Hungary. Email: nemes.attila@med.u-szeged.hu.
}

\begin{abstract}
Background: Aortopathy is a common phenomenon in tetralogy of Fallot (TOF). The current study was designed to detect left ventricular (LV) deformation abnormalities and its relation to aortic stiffness in corrected TOF (cTOF) using the novel three-dimensional (3D) speckle-tracking echocardiography (3DSTE). Detailed comparative analysis between patients with early palliation-late correction (pcTOF) and early total reconstruction (etrTOF) was also performed.

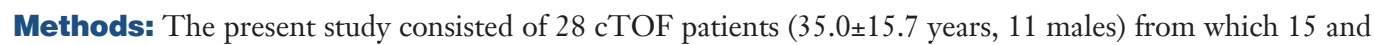
13 proved to be pcTOF and etrTOF, respectively. Their clinical parameters were compared to those of 39 matched healthy adults $(35.5 \pm 6.0$ years, 16 males).

Results: cTOF patients showed significantly lower global LV longitudinal, circumferential and area strains as compared to controls. In etrTOF patients, global LV 3D strain was higher than in controls. In pcTOF patients, all LV strains proved to be significantly lower as compared to those of etrTOF patients and controls. In all cTOF patients, several moderate correlations could be detected between LV strain parameters and aortic elastic properties.

Conclusions: Significant LV deformational abnormalities could be demonstrated in cTOF patients. etrTOF patients have beneficial LV strain parameters as compared to those of pcTOF patients. LV strains show correlations with aortic elastic properties.
\end{abstract}

Keywords: Fallot; aortic stiffness; three-dimensional (3D); speckle-tracking; echocardiography; strain

Submitted Mar 17, 2020. Accepted for publication May 19, 2020.

doi: $10.21037 / \mathrm{cdt}-20-366$

View this article at: http://dx.doi.org/10.21037/cdt-20-366

\section{Introduction}

Aortopathy is a common phenomenon associated with congenital heart disease (CHD). The current literature considers coarctation of the aorta, bicuspid aortic valve, tetralogy of Fallot (TOF), pulmonary atresia with ventricular septal defect (VSD) and truncus arteriosus to be the most important patient groups regarding the development of aortopathy, apart from genetic syndromes and secondary aortopathy is often seen after surgical repair including the Ross operation (1). TOF is considered to be the most common cyanotic CHD, which is described as the co-occurrence of pulmonary stenosis, VSD, right ventricular (RV) hypertrophy and for the current study most importantly overriding aorta (2). Aortic dilation in TOF is widely described, and has been formerly associated with volume overload due to haemodynamic alterations 
in unrepaired TOF (1). However, it is increasingly discussed that even late after surgical correction, abnormal dimensions and impaired elastic properties may be found in the ascending aorta in corrected TOF (cTOF) (3). Further histopathologic studies have confirmed that there are intrinsic abnormalities in the aortic wall, which may be the cause of progressive aortic dilation and elastic abnormalities (4). In recent two-dimensional (2D) speckletracking echocardiographic (STE) studies, during left ventricular (LV) strain analyses significant abnormalities in $\mathrm{LV}$ deformation could be detected in cTOF (5). It is known that strong relationship exists between aortic and $\mathrm{LV}$ function called aorto-ventricular coupling. According to recent findings, aortopathy is thought to negatively influence LV function in CHD (6).

TOF patients are generally classified in two distinct subgroups regarding surgical strategies. Either early palliative surgery (creating artificial left-to-right shunts to provide adequate pulmonary blood flow) is performed first, with late total reconstruction (pcTOF), or an early total reconstruction (etrTOF) is performed (7). The present study was designed to detect subtle alterations of $\mathrm{LV}$ deformation in cTOF using the novel three-dimensional speckle-tracking echocardiography (3DSTE). Detailed comparative analysis of $\mathrm{LV}$ deformation between pcTOF and etrTOF patients was also aimed to be performed. It was also purposed to find any correlation between LV deformation and aortic elastic properties in the subgroup of cTOF patients. We present the following article in accordance with the STROBE reporting checklist (available at http://dx.doi.org/10.21037/cdt-20-366).

\section{Methods}

\section{Patients}

In our department a registry was created to collect data of CHD patients treated and/or operated on at the Department of Pediatrics, Department of Heart Surgery, and 2nd Department of Medicine and Cardiology Center at the University of Szeged, Hungary since 1961. This is called the Registry of C(S)ONGenital caRdiAc Disease patients at the University of Szeged (CSONGRAD Registry), which involves data of more than 3,000 CHD patients (8). Twentyeight adult cTOF patients were involved in the present study who were followed-up with the help of CSONGRAD Registry, and who had repair at the age of $6.04 \pm 3.83$ years. Of these patients, 15 underwent early palliative surgery
[Blalock-Taussig ( $\mathrm{n}=10)$, Waterston-Cooley $(\mathrm{n}=3)$ shunts, or Brock procedure $(n=2)$ ] and late total correction (pcTOF), while early total reconstruction was the treatment of choice in 13 cTOF patients (etrTOF). Their clinical parameters were compared to those of 39 age- and gender-matched healthy adults. All patients and controls underwent routine transthoracic 2D Doppler echocardiography extended with 3DSTE. The present work is part of the MAGYAR-Path Study (Motion Analysis of the heart and Great vessels bY three-dimensionAl speckle-tRacking echocardiography in Pathological cases), which was organized partly to examine CHD-related 3DSTE-derived parameters. All cTOF patients and control subjects gave informed consent. The institutional human research committee approved the study which complied with the 1975 Declaration of Helsinki (registration number: 71/2011).

\section{Transthoracic echocardiography}

Echocardiographic examinations were performed with a Toshiba Artida ${ }^{\mathrm{TM}}$ cardiac ultrasound system (Toshiba Medical Systems, Tokyo, Japan) with a PST-30SBP 1-5 MHz phased-array transducer. The echocardiographic studies were stored digitally, and underwent offline analysis (Á Kormányos, P Domsik). The experts who performed the analysis were unaware of the clinical data. Left heart dimensions and parameters, including LV diameters, volumes, ejection fraction (EF), aortic and left atrial (LA) sizes were all measured from parasternal long-axis view using M-mode echocardiography (9). These measurements were derived and averaged from 3 cardiac cycles.

\section{Measurement of blood pressure values}

After 10 minutes of rest, patients were placed in a supine position, and with the cuff applied to the right arm, systolic and diastolic blood pressures (SBP and DBP, respectively) were measured. The first and fifth Korotkoff sounds were considered to indicate SBP and DBP. Patients did not consume caffeinated drinks or cigarettes before blood pressure measurements (10).

\section{Evaluation of aortic stiffness parameters}

During standard transthoracic echocardiographic examination, ascending aortic diameters were recorded in parasternal long-axis view with $\mathrm{M}$-mode $3 \mathrm{~cm}$ above the level of the aortic valve. Systolic and diastolic aortic 


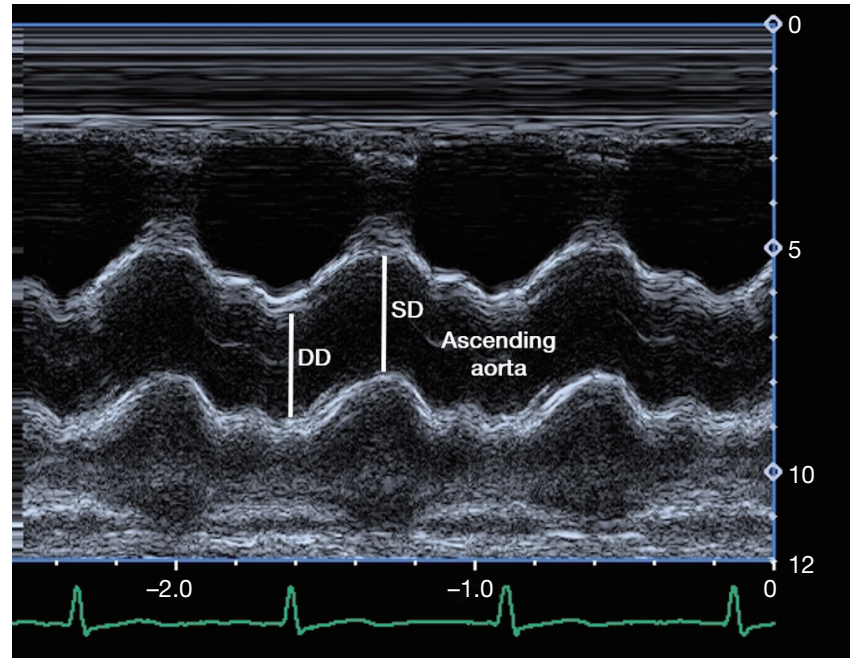

Figure 1 Measurements of systolic (SD) and diastolic (DD) diameters of the ascending aorta are presented in an M-mode echocardiographic image obtained $3 \mathrm{~cm}$ above the aortic valve.

diameters (SD and DD) were measured at the maximum of the anterior motion of the aorta, and the peak of the QRS (Figure 1). Measurements were repeated 3 times and averaged. Aortic elastic properties have been calculated with the formulae bellow (10):

* Aortic strain $(\mathrm{AS})=(\mathrm{SD}-\mathrm{DD}) / \mathrm{DD}$;

* Aortic distensibility $(\mathrm{AD})=2 \times(\mathrm{SD}-\mathrm{DD}) /[(\mathrm{SBP}-$ DBP) $\times$ DD];

- Aortic stiffness index $(\mathrm{ASI})=\ln (\mathrm{SBP} / \mathrm{DBP}) /[(\mathrm{SD}$ $\mathrm{DD}) / \mathrm{DD}$ ] ("ln" is the natural logarithm).

\section{DSTE}

3DSTE studies were also performed with the same Toshiba Artida $^{\mathrm{TM}}$ echocardiography system (Toshiba, Tokyo, Japan) using a 1-4 MHz matrix phased-array PST-25SX transducer placed in an apical window. The full pyramid-shaped dataset was created from 6 wedge-shaped subvolumes acquired with RR interval monitored by electrocardiogram (ECG) if remained the same. Quantification was performed using 3D Wall Motion Tracking software version 2.7 (Toshiba Medical Systems). At end-diastole, apical two- (AP2CH) and four-chamber $(\mathrm{AP} 4 \mathrm{CH})$ views and 3 short-axis views at different LV levels were automatically reconstructed from the acquired 3D echocardiographic cloud. The region of interest was then manually marked on the AP2CH and $\mathrm{AP} 4 \mathrm{CH}$ views, with two points at the bases of the $\mathrm{LV}$ at the edges of the mitral annulus, and one at the endocardial surface of the LV apex (Figure 2). The reconstructed LV endocardial surface was then tracked during the cardiac cycle. 3DSTE-derived global and segmental LV radial (RS), circumferential (CS), longitudinal (LS), 3D (3DS) and area (AS) strains were generated by the software (11).

\section{Statistical analysis}

Continuous variables are reported as mean values \pm standard deviation, while categorical data are summarized as frequencies and percentages. Comparisons were performed with Student $t$-test, $\chi^{2}$ test, and Fisher's exact test, when appropriate. Statistical significance was considered when the $\mathrm{p}$ value proved to be less than 0.05 . Pearson's formula was used to determine numerical correlations. For statistical analysis Medcalc software (MedCalc, Mariakerke, Belgium) was used.

\section{Results}

\section{Clinical and demographic data}

Patient and control groups were age- and gender-matched. There was no statistically significant difference in the presence of co-morbidities except for hypertension which was found in almost one third of cTOF patients. Body surface area was comparable in the patient and control groups (Table 1). Significant (> grade 2) tricuspid regurgitations could be detected in $4(15 \%)$ cTOF patients. None of the cTOF patients showed significant mitral regurgitation.

\section{D echocardiographic parameters}

Of the standard 2D echocardiographic parameters, LA diameter proved to be significantly larger in cTOF patients regardless of the treatment method. $\mathrm{LV}$ dimensions and function (except for $\mathrm{LV}$ end-systolic diameter) did not differ significantly between the groups examined (Table 1).

\section{Aortic stiffness parameters}

Aortic dimensions were somewhat dilated, $\mathrm{AS}$ and $\mathrm{AD}$ were reduced, while ASI proved to be increased in cTOF patients as compared to controls. When patients with different treatments were compared, etrTOF patients showed more beneficial aortic elastic properties compared to those of pcTOF patients. 


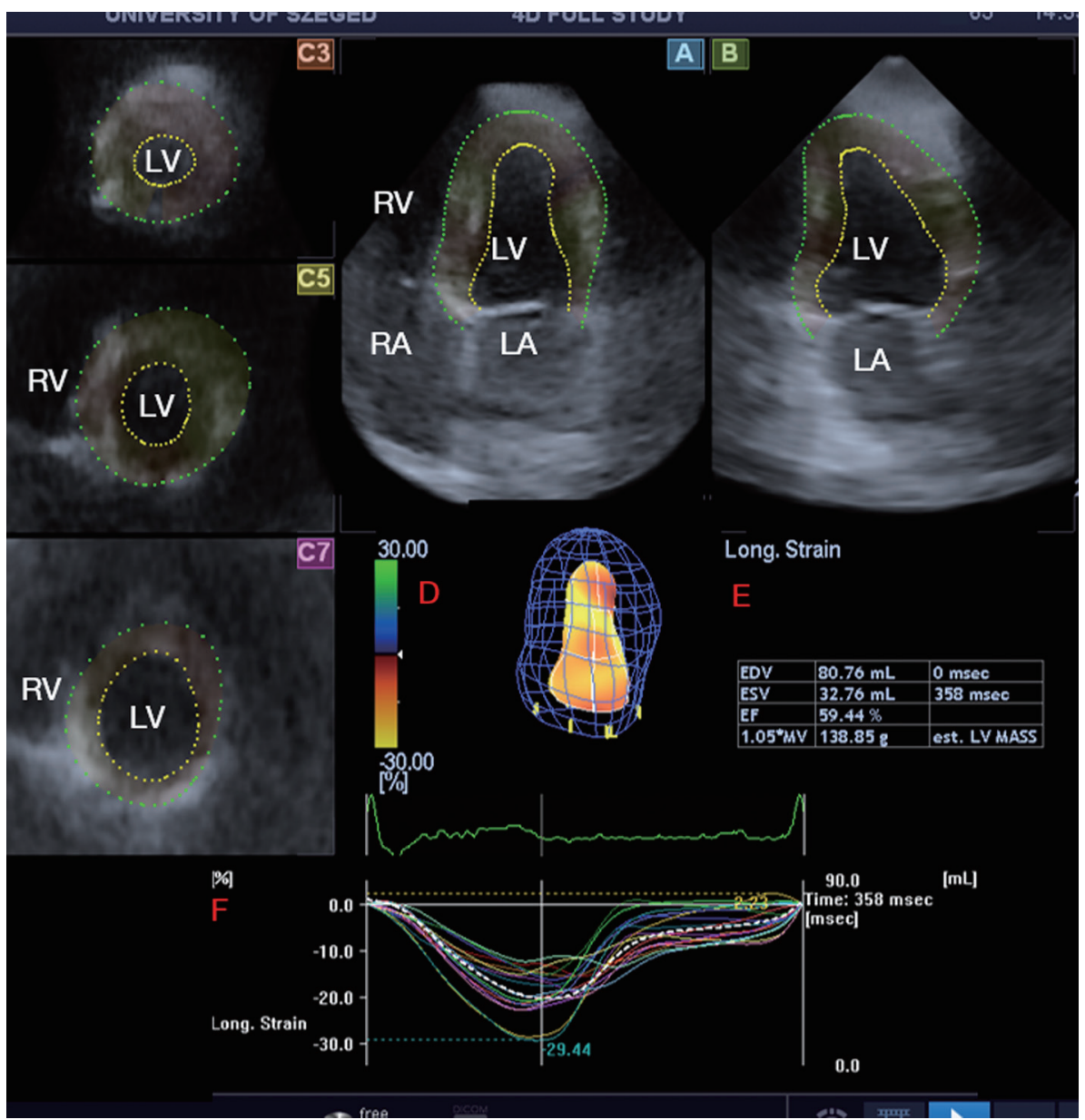

Figure 2 Left ventricular (LV) three-dimensional (3D) speckle-tracking echocardiographic analysis of a patient with corrected tetralogy of Fallot. The following views are presented: (A) apical four-chamber view, (B) apical two-chamber view, (C3) short-axis view at basal, (C5) mid- and (C7) apical LV level together with 3D model of the LV (D) and LV volumetric data (E). Colored curves represent time-LV segmental strains. LA, left atrium; LV, left ventricle; RA, right atrium; RV, right ventricle; EDV, end-diastolic volume; ESV, end-systolic volume; EF, ejection fraction.

\section{DSTE-derived LV volumetric data}

For all cTOF patients 3DSTE-derived LV end-systolic volume (ESV) was higher and LV-EF proved to be lower as compared to controls. Subgroup analysis showed that pcTOF patients had significantly larger LV-ESV and lower LV-EF than controls and etrTOF patients (Table 2).

\section{DSTE-derived LV strains}

cTOF patients showed significantly lower global and mean segmental LV-LS, LV-CS and LV-AS as compared to controls. In etrTOF patients global LV-3DS was higher than in controls. In pcTOF patients all global and mean segmental LV strains proved to be significantly lower as compared to those of etrTOF patients (Table 2).

Segmental LV-LS, LV-CS, LV-RS, LV-AS and LV-3DS parameters are presented in Tables 3-7. Most segmental $\mathrm{LV}$ strains of pcTOF patients were significantly lower as compared to those of etrTOF patients and controls.

\section{Correlations}

Correlation coefficients between 3DSTE-derived strains and aortic elastic properties are demonstrated in Table 8. In all cTOF patients, several moderate correlations could be detected between LV strain parameters and aortic 
Table 1 Comparison of baseline demographic, two-dimensional echocardiographic data and aortic elastic properties in different group of patients with tetralogy of Fallot and in controls

\begin{tabular}{|c|c|c|c|c|}
\hline Data & Controls $(n=39)$ & $\begin{array}{l}\text { All cTOF patients } \\
\qquad(n=28)\end{array}$ & $\begin{array}{l}\text { etrTOF patients } \\
\qquad(n=13)\end{array}$ & $\begin{array}{c}\text { pcTOF patients } \\
\quad(n=15)\end{array}$ \\
\hline \multicolumn{5}{|l|}{ Risk factors } \\
\hline Female gender [\%] & $16[41]$ & $11[39]$ & $7[54]$ & $4[27]$ \\
\hline Hypercholesterolaemia [\%] & $0[0]$ & $1[4]$ & $1[8]$ & $0[0]$ \\
\hline Body surface area $\left(\mathrm{m}^{2}\right)$ & $1.90 \pm 0.28$ & $1.81 \pm 0.26$ & $1.89 \pm 0.23$ & $1.72 \pm 0.26$ \\
\hline \multicolumn{5}{|l|}{ Two-dimensional echocardiographic data } \\
\hline LV end-systolic diameter (mm) & $33.0 \pm 9.2$ & $31.5 \pm 6.4^{*}$ & $31.2 \pm 5.3$ & $31.7 \pm 7.6$ \\
\hline LV end-systolic volume (mL) & $36.6 \pm 9.0$ & $42.3 \pm 26.9$ & $39.5 \pm 20.3$ & $44.8 \pm 32.5$ \\
\hline Interventricular septum (mm) & $9.2 \pm 1.5$ & $9.7 \pm 1.5$ & $9.5 \pm 1.1$ & $9.9 \pm 1.8$ \\
\hline LV posterior wall (mm) & $9.4 \pm 1.8$ & $9.5 \pm 1.4$ & $9.4 \pm 1.4$ & $9.6 \pm 1.5$ \\
\hline LV ejection fraction (\%) & $64.2 \pm 3.5$ & $63.9 \pm 11.2$ & $65.5 \pm 6.1$ & $62.4 \pm 14.5$ \\
\hline \multicolumn{5}{|l|}{ Aortic stiffness parameters } \\
\hline Systolic aortic diameter (mm) & $29.5 \pm 3.9$ & $31.5 \pm 5.1$ & $31.4 \pm 5.2$ & $31.6 \pm 5.1$ \\
\hline Aortic stiffness index & $6.18 \pm 5.58$ & $12.95 \pm 11.25^{\star}$ & $8.57 \pm 5.38$ & $16.46 \pm 13.52^{\star \dagger}$ \\
\hline
\end{tabular}

${ }^{*}, \mathrm{P}<0.05$ vs. controls; ${ }^{\dagger}, \mathrm{P}<0.05$ vs. pcTOF. cTOF, corrected tetralogy of Fallot; etrTOF, early total reconstruction for patients with TOF; pcTOF, early palliation; late correction for patients with TOF; LA, left atrial; LV, left ventricular.

elastic properties. The subgroup analysis showed moderate correlations between LV-CS, LV-LS and LV-AS and AD in etrTOF patients. In pcTOF patients, only LV-RS correlated with aortic strain and LV-CS with ASI.

\section{Discussion}

To the best of the authors' knowledge, the relationship between 3DSTE-derived LV strains and aortic elastic properties has not been simultaneously examined, and their correlation has never been investigated in cTOF patients. Theoretically cardiovascular risk factors could have effects on LV deformation and aortic size and function at the same time. However, there are interactions between the LV and the arterial system as well called ventricular-arterial coupling, the LV and the arterial system has effects on each other. In recent studies, aortic elasticity properties showed correlations with LV-CS and LV rotational parameters even in healthy subjects $(12,13)$. It has already been demonstrated that $\mathrm{CTOF}$ - even with preserved LV-EF_-exhibits reduced 
Table 2 Comparison of three-dimensional speckle-tracking echocardiography-derived left ventricular volumetric parameters, global and mean segmental strains in different group of patients with tetralogy of Fallot and in controls

\begin{tabular}{|c|c|c|c|c|}
\hline Data & Controls $(n=39)$ & All cTOF patients $(n=28)$ & etrTOF patients $(n=13)$ & pcTOF patients $(n=15)$ \\
\hline LV-EDV (mL) & $84.8 \pm 24.7$ & $95.6 \pm 35.8$ & $93.3 \pm 36.8$ & $97.6 \pm 36.2$ \\
\hline LV-ESV (mL) & $35.6 \pm 15.5$ & $53.6 \pm 33.7^{\star}$ & $46.4 \pm 34.1$ & $59.8 \pm 33.2^{\star \dagger}$ \\
\hline LV-EF (\%) & $57.9 \pm 5.5$ & $46.9 \pm 14.1^{*}$ & $53.7 \pm 11.5$ & $41.0 \pm 13.8^{\star \dagger}$ \\
\hline RS (\%) & $25.2 \pm 10.4$ & $23.1 \pm 13.0$ & $30.2 \pm 10.6$ & $17.0 \pm 11.9^{\star \dagger}$ \\
\hline CS (\%) & $-27.6 \pm 4.9$ & $-20.7 \pm 9.1^{*}$ & $-24.7 \pm 8.7$ & $-17.3 \pm 8.2^{\star \dagger}$ \\
\hline LS (\%) & $-17.1 \pm 2.5$ & $-14.8 \pm 4.0^{*}$ & $-15.6 \pm 3.2$ & $-12.1 \pm 4.7^{\star \dagger}$ \\
\hline 3DS (\%) & $27.7 \pm 10.1$ & $25.5 \pm 12.6$ & $32.7 \pm 10.2^{*}$ & $19.3 \pm 11.4^{\star^{\dagger}}$ \\
\hline RS (\%) & $27.4 \pm 10.4$ & $26.9 \pm 12.7$ & $33.8 \pm 10.1^{*}$ & $20.9 \pm 11.7^{\star^{\dagger}}$ \\
\hline CS (\%) & $-28.5 \pm 5.0$ & $-22.3 \pm 8.5^{\star}$ & $-26.4 \pm 7.9$ & $-18.7 \pm 7.6^{\star \dagger}$ \\
\hline LS (\%) & $-17.1 \pm 2.5$ & $-14.8 \pm 4.0^{*}$ & $-16.8 \pm 2.4$ & $-13.1 \pm 4.5^{\star \dagger}$ \\
\hline 3DS (\%) & $29.7 \pm 10.0$ & $28.7 \pm 12.6$ & $35.9 \pm 10.0^{*}$ & $22.4 \pm 11.2^{\star^{\dagger}}$ \\
\hline AS (\%) & $-41.4 \pm 4.9$ & $-33.2 \pm 10.6^{*}$ & $-38.4 \pm 8.6$ & $-28.7 \pm 10.4^{\star \dagger}$ \\
\hline
\end{tabular}

${ }^{*}, \mathrm{P}<0.05$ vs. controls; ${ }^{\dagger}, \mathrm{P}<0.05$ vs. etrTOF. cTOF, corrected tetralogy of Fallot; etrTOF, early total reconstruction for patients with TOF; pcTOF, early palliation, late correction for patients with TOF; LV, left ventricular; 3DSTE, three-dimensional speckle-tracking echocardiography; RS, radial strain; CS, circumferential strain; LS, longitudinal strain; 3DS, three-dimensional strain; AS, area strain.

Table 3 Comparison of three-dimensional speckle-tracking echocardiography-derived left ventricular segmental radial strains in different group of patients with tetralogy of Fallot and in controls

\begin{tabular}{lcccc}
\hline Data & Controls $(n=39)$ & All cTOF $(n=28)$ & etrTOF $(n=13)$ & pcTOF $(n=15)$ \\
\hline BA (\%) & $35.9 \pm 22.8$ & $31.0 \pm 29.4$ & $38.7 \pm 36.2$ & $24.3 \pm 21.0$ \\
BAS (\%) & $39.6 \pm 20.3$ & $31.7 \pm 22.3^{\star}$ & $37.4 \pm 28.1$ & $26.7 \pm 15.1^{*}$ \\
BIS (\%) & $28.6 \pm 14.1$ & $30.2 \pm 20.2$ & $33.1 \pm 20.1$ & $27.7 \pm 20.7$ \\
BI (\%) & $25.1 \pm 16.3$ & $25.1 \pm 18.0$ & $28.5 \pm 17.6$ & $22.1 \pm 18.4$ \\
BIL (\%) & $29.7 \pm 21.4$ & $21.8 \pm 13.9$ & $24.6 \pm 15.6$ & $19.3 \pm 12.3$ \\
BAL (\%) & $31.1 \pm 22.9$ & $26.7 \pm 27.8$ & $33.3 \pm 33.0$ & $20.9 \pm 22.0$ \\
MA (\%) & $31.1 \pm 18.1$ & $32.5 \pm 28.1$ & $41.7 \pm 28.2$ & $24.5 \pm 26.4^{\dagger}$ \\
MAS (\%) & $34.6 \pm 15.2$ & $30.5 \pm 20.2$ & $39.8 \pm 21.2$ & $22.5 \pm 15.9^{* \dagger}$ \\
MIS (\%) & $27.6 \pm 14.0$ & $31.1 \pm 19.1$ & $38.5 \pm 15.4^{*}$ & $24.7 \pm 20.1$ \\
MI (\%) & $26.4 \pm 17.8$ & $29.1 \pm 23.3$ & $40.9 \pm 27.1^{*}$ & $19.0 \pm 13.3^{\dagger}$ \\
MIL (\%) & $29.6 \pm 21.9$ & $29.4 \pm 24.3$ & $42.3 \pm 26.6$ & \\
MAL (\%) & $27.9 \pm 18.2$ & $33.7 \pm 32.0$ & $46.4 \pm 31.6$ & $22.7 \pm 28.9^{\dagger}$ \\
\hline
\end{tabular}

Table 3 (continued) 
Table 3 (continued)

\begin{tabular}{lcccc}
\hline Data & Controls $(n=39)$ & All cTOF $(n=28)$ & etrTOF $(n=13)$ & pcTOF $(n=15)$ \\
\hline AA (\%) & $16.6 \pm 11.4$ & $16.9 \pm 12.1$ & $18.7 \pm 12.5$ & $15.3 \pm 11.9$ \\
AS (\%) & $19.2 \pm 11.9$ & $21.2 \pm 12.3$ & $26.5 \pm 10.4^{*}$ & $16.7 \pm 12.2^{\dagger}$ \\
Al (\%) & $19.6 \pm 15.5$ & $19.5 \pm 19.6$ & $27.6 \pm 22.1$ & $12.5 \pm 14.4$ \\
AL (\%) & $16.5 \pm 11.4$ & $19.9 \pm 22.5$ & $23.3 \pm 22.6$ & $17.0 \pm 22.8$ \\
\hline
\end{tabular}

${ }^{*}, \mathrm{P}<0.05$ vs. controls; ${ }^{\dagger}, \mathrm{P}<0.05$ vs. etrTOF. cTOF, corrected tetralogy of Fallot; etrTOF, early total reconstruction for patients with TOF; pcTOF, early palliation, late correction for patients with TOF; BA, basal anterior; BAS, basal anteroseptal; BIS, basal inferoseptal; BI, basal inferior; BIL, basal inferolateral; BAL, basal anterolateral; MA, midventricular anterior; MAS, midventricular anteroseptal; MIS, midventricular inferoseptal; MI, midventricular inferior; MIL, midventricular inferolateral; MAL, midventricular anterolateral; AA, apical anterior. AS, apical septal; Al, apical inferior; AL, apical lateral.

Table 4 Comparison of three-dimensional speckle-tracking echocardiography-derived left ventricular segmental circumferential strains in different group of patients with tetralogy of Fallot and in controls

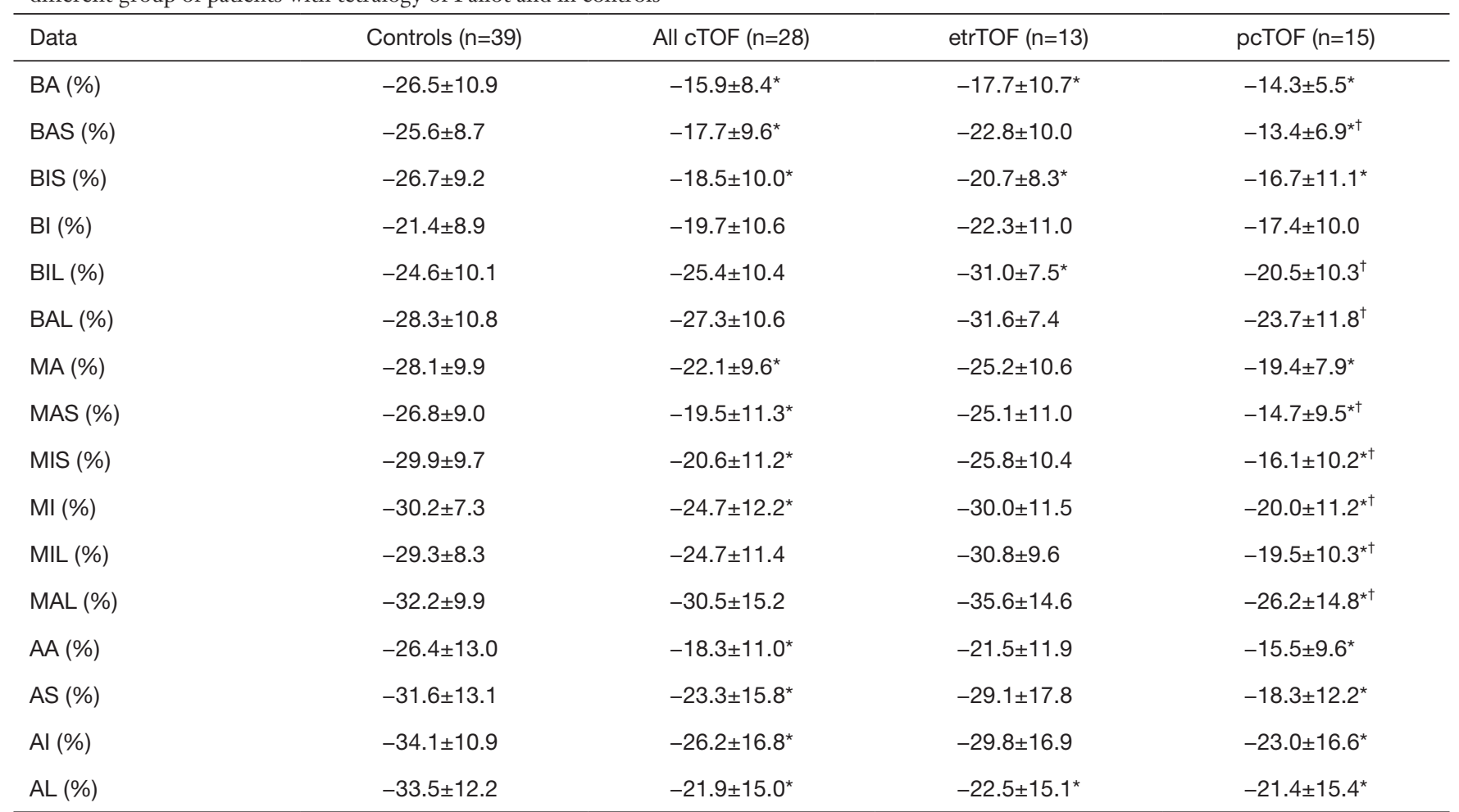

${ }^{*}, \mathrm{P}<0.05$ vs. controls; ${ }^{\dagger}, \mathrm{P}<0.05$ vs. etrTOF patients. cTOF, corrected tetralogy of Fallot; etrToF, early total reconstruction for patients with TOF; pcTOF, early palliation, late correction for patients with TOF; BA, basal anterior; BAS, basal anteroseptal; BIS, basal inferoseptal; $\mathrm{BI}$, basal inferior; BIL, basal inferolateral; BAL, basal anterolateral; MA, midventricular anterior; MAS, midventricular anteroseptal; MIS, midventricular inferoseptal; MI, midventricular inferior; MIL, midventricular inferolateral; MAL, midventricular anterolateral; AA, apical anterior. AS, apical septal; Al, apical inferior; AL, apical lateral.

global LV-LS and LV-CS measured by 2DSTE. This suggests that there is an underlying subclinical LV damage even after surgical repair (5). Furthermore, it has also been found that abnormal global LV-LS and RV-LS and LV rotation are associated with adverse cardiovascular events, highlighting the potential clinical usefulness of STEderived parameters determining prognosis, and finding which patients are at high risk (14). Aortic dilation is also a known abnormality in cTOF, which is associated with higher ASI (3). This was confirmed by both M-mode 
Table 5 Comparison of three-dimensional speckle-tracking echocardiography-derived left ventricular segmental longitudinal strains in different group of patients with tetralogy of Fallot and in controls

\begin{tabular}{|c|c|c|c|c|}
\hline Data & Controls $(n=39)$ & All cTOF $(n=28)$ & etrTOF $(n=13)$ & pcTOF $(n=15)$ \\
\hline BAS (\%) & $-18.1 \pm 5.9$ & $-12.2 \pm 4.9^{\star}$ & $-13.6 \pm 4.1^{*}$ & $-11.0 \pm 5.4^{*}$ \\
\hline BIS (\%) & $-15.9 \pm 4.2$ & $-15.8 \pm 6.1$ & $-17.4 \pm 4.7$ & $-14.4 \pm 6.9$ \\
\hline $\mathrm{BI}(\%)$ & $-16.8 \pm 4.7$ & $-18.7 \pm 6.5$ & $-20.7 \pm 6.6$ & $-16.9 \pm 6.0$ \\
\hline BAL (\%) & $-25.0 \pm 7.8$ & $-19.4 \pm 7.4^{*}$ & $-22.6 \pm 6.2$ & $-16.6 \pm 7.3^{\star^{\dagger}}$ \\
\hline MA (\%) & $-11.9 \pm 6.9$ & $-15.3 \pm 7.1^{*}$ & $-17.4 \pm 7.2^{*}$ & $13.4 \pm 6.7$ \\
\hline MAS (\%) & $-14.2 \pm 4.6$ & $-11.9 \pm 5.2$ & $-14.4 \pm 3.5$ & $-9.7 \pm 5.5^{\star \dagger}$ \\
\hline MIS (\%) & $-14.7 \pm 4.0$ & $-12.1 \pm 5.7$ & $-14.4 \pm 5.8$ & $-10.1 \pm 5.1^{*}$ \\
\hline MAL (\%) & $-14.3 \pm 6.1$ & $-15.6 \pm 7.8$ & $-18.5 \pm 7.5$ & $-13.1 \pm 7.4^{\dagger}$ \\
\hline AA (\%) & $-10.9 \pm 7.6$ & $-8.4 \pm 6.5$ & $-9.2 \pm 7.6$ & $-7.7 \pm 5.7$ \\
\hline AS (\%) & $-24.4 \pm 9.4$ & $-11.6 \pm 7.6^{*}$ & $-13.7 \pm 7.2^{*}$ & $-9.8 \pm 7.7^{\star}$ \\
\hline $\mathrm{Al}(\%)$ & $-23.6 \pm 8.2$ & $-19.5 \pm 11.8$ & $-20.6 \pm 11.7$ & $-18.6 \pm 12.3$ \\
\hline AL (\%) & $-11.6 \pm 7.0$ & $-17.2 \pm 10.4^{*}$ & $-16.4 \pm 8.6$ & $-17.9 \pm 12.1$ \\
\hline
\end{tabular}

${ }^{*}, \mathrm{P}<0.05$ vs. controls; ${ }^{\dagger}, \mathrm{P}<0.05$ vs. etrTOF patients. cTOF, corrected tetralogy of Fallot; etrToF, early total reconstruction for patients with TOF; pcTOF, early palliation, late correction for patients with TOF; BA, basal anterior; BAS, basal anteroseptal; BIS, basal inferoseptal; $\mathrm{BI}$, basal inferior; BIL, basal inferolateral; BAL, basal anterolateral; MA, midventricular anterior; MAS, midventricular anteroseptal; MIS, midventricular inferoseptal; MI, midventricular inferior; MIL, midventricular inferolateral; MAL, midventricular anterolateral; AA, apical anterior. AS, apical septal; Al, apical inferior; AL, apical lateral.

Table 6 Comparison of three-dimensional speckle-tracking echocardiography-derived left ventricular segmental area strains in different group of patients with tetralogy of Fallot and in controls

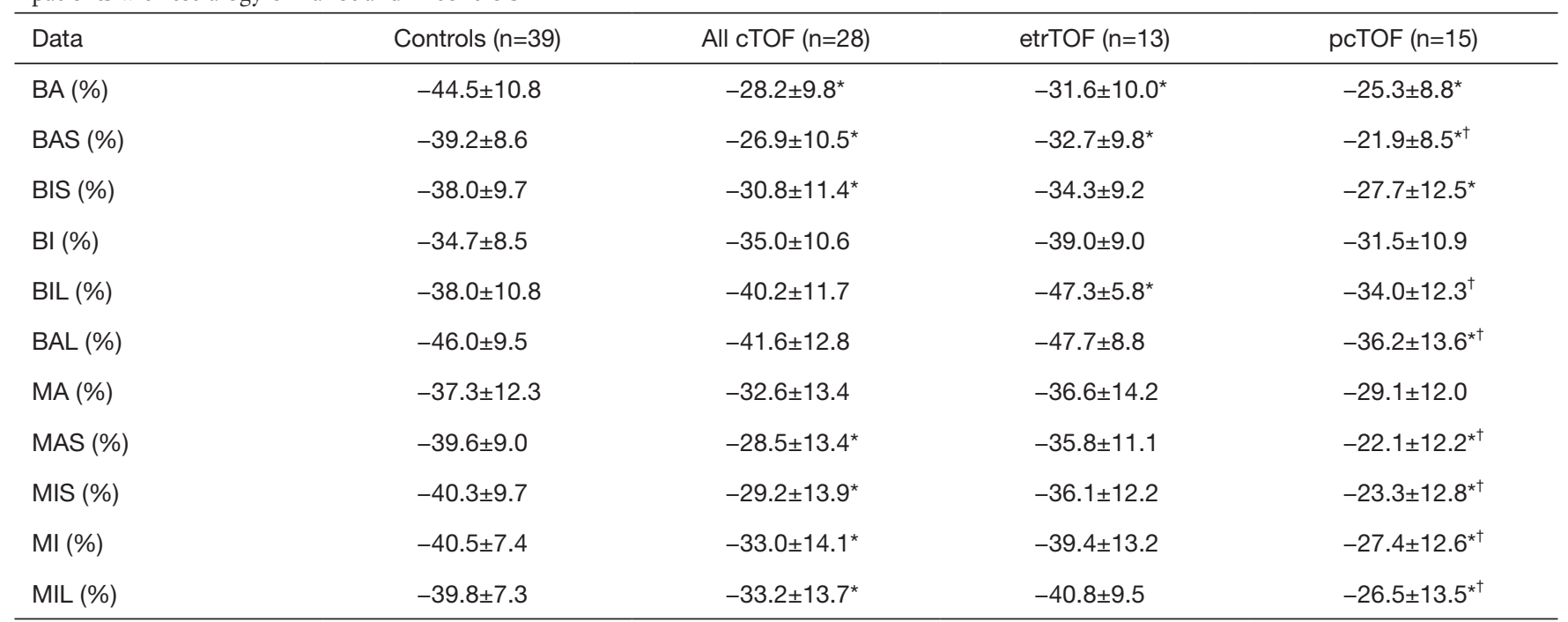

Table 6 (continued) 
Table 6 (continued)

\begin{tabular}{|c|c|c|c|c|}
\hline Data & Controls $(n=39)$ & All cTOF $(n=28)$ & etrTOF $(n=13)$ & pcTOF $(n=15)$ \\
\hline AA (\%) & $-36.4 \pm 15.0$ & $-25.0 \pm 13.9^{*}$ & $-28.3 \pm 14.8$ & $-22.2 \pm 13.0^{*}$ \\
\hline AS (\%) & $-49.4 \pm 14.4$ & $-32.0 \pm 19.0^{*}$ & $-38.8 \pm 20.2$ & $-26.1 \pm 16.4^{*}$ \\
\hline $\mathrm{Al}(\%)$ & $-49.9 \pm 11.3$ & $-39.3 \pm 22.0$ & $-43.3 \pm 20.9$ & $-35.9 \pm 23.1^{*}$ \\
\hline
\end{tabular}

${ }^{*}, \mathrm{P}<0.05$ vs. controls; ${ }^{\dagger}, \mathrm{P}<0.05$ vs. etrTOF patients. cTOF, corrected tetralogy of Fallot; etrToF, early total reconstruction for patients with TOF; pcTOF, early palliation, late correction for patients with TOF; BA, basal anterior; BAS, basal anteroseptal; BIS, basal inferoseptal; $\mathrm{BI}$, basal inferior; BIL, basal inferolateral; BAL, basal anterolateral; MA, midventricular anterior; MAS, midventricular anteroseptal; MIS, midventricular inferoseptal; MI, midventricular inferior; MIL, midventricular inferolateral; MAL, midventricular anterolateral; AA, apical anterior. AS, apical septal; Al, apical inferior; AL, apical lateral.

Table 7 Comparison of three-dimensional speckle-tracking echocardiography-derived left ventricular segmental three-dimensional strains in different group of patients with tetralogy of Fallot and in controls

\begin{tabular}{|c|c|c|c|c|}
\hline Data & Controls $(n=39)$ & All cTOF $(n=28)$ & etrTOF $(n=13)$ & pcTOF $(n=15)$ \\
\hline BAS (\%) & $40.4 \pm 20.3$ & $32.9 \pm 22.0$ & $38.6 \pm 27.4$ & $27.9 \pm 15.4^{*}$ \\
\hline BIS (\%) & $30.1 \pm 14.3$ & $31.6 \pm 18.8$ & $34.9 \pm 18.9$ & $28.7 \pm 18.8$ \\
\hline $\mathrm{BI}(\%)$ & $28.8 \pm 14.8$ & $28.1 \pm 17.6$ & $32.3 \pm 15.2$ & $24.5 \pm 19.3$ \\
\hline BAL (\%) & $34.0 \pm 21.2$ & $30.2 \pm 27.5$ & $37.7 \pm 33.5$ & $23.7 \pm 20.1$ \\
\hline MA (\%) & $31.7 \pm 18.0$ & $33.5 \pm 26.5$ & $43.2 \pm 27.1$ & $25.2 \pm 23.8$ \\
\hline MAS (\%) & $35.4 \pm 14.9$ & $31.8 \pm 19.5$ & $41.1 \pm 19.6$ & $23.8 \pm 16.1^{\star \dagger}$ \\
\hline MIS (\%) & $28.9 \pm 13.9$ & $32.1 \pm 17.9$ & $39.7 \pm 14.3^{*}$ & $25.4 \pm 18.5^{\dagger}$ \\
\hline AA (\%) & $17.5 \pm 11.6$ & $18.4 \pm 12.3$ & $20.7 \pm 12.2$ & $16.4 \pm 12.4$ \\
\hline AS (\%) & $21.0 \pm 12.3$ & $23.2 \pm 11.9$ & $29.0 \pm 10.0^{*}$ & $18.2 \pm 11.5^{\dagger}$ \\
\hline $\mathrm{Al}(\%)$ & $21.2 \pm 15.5$ & $20.7 \pm 19.2$ & $29.4 \pm 21.7$ & $13.1 \pm 13.2^{\dagger}$ \\
\hline AL (\%) & $18.7 \pm 12.1$ & $21.0 \pm 22.0$ & $24.9 \pm 23.2$ & $17.7 \pm 21.1$ \\
\hline
\end{tabular}

${ }^{*}, \mathrm{P}<0.05$ vs. controls; ${ }^{\dagger}, \mathrm{P}<0.05$ vs. etrTOF patients. cTOF, corrected tetralogy of Fallot; etrToF, early total reconstruction for patients with TOF; pcTOF, early palliation, late correction for patients with TOF; BA, basal anterior; BAS, basal anteroseptal; BIS, basal inferoseptal; $\mathrm{BI}$, basal inferior; BIL, basal inferolateral; BAL, basal anterolateral; MA, midventricular anterior; MAS, midventricular anteroseptal; MIS, midventricular inferoseptal; MI, midventricular inferior; MIL, midventricular inferolateral; MAL, midventricular anterolateral; AA, apical anterior; AS, apical septal; Al, apical inferior; AL, apical lateral.

echocardiography- and novel 2DSTE-derived aortic stiffness parameters (15).

Our results confirm previous studies describing that cTOF is associated with impaired LV deformations $(16,17)$.
Importantly, not only the widely used 2DSTE-derived LV-LS proved to be the most robust. LV-AS, and most notably LV-CS are significantly impaired in cTOF patients, highlighting the clinical significance of 3DSTE having the 
Table 8 Correlations between aortic elastic properties and left ventricular global strains in different group of patients with tetralogy of Fallot and in controls

\begin{tabular}{|c|c|c|c|}
\hline Data & Aortic strain & Aortic distensibility & Aortic stiffness index \\
\hline \multicolumn{4}{|c|}{ In all cTOF patients } \\
\hline Global LV-RS & $0.447^{\star}$ & 0.327 & $-0.417^{*}$ \\
\hline Global LV-CS & $-0.416^{\star}$ & $-0.477^{\star}$ & $0.449^{*}$ \\
\hline Global LV-LS & -0.339 & -0.339 & 0.354 \\
\hline Global LV-3DS & $0.456^{\star}$ & 0.344 & $-0.433^{\star}$ \\
\hline Global LV-AS & $-0.395^{\star}$ & $-0.427^{*}$ & $0.422^{\star}$ \\
\hline \multicolumn{4}{|l|}{ In etrTOF patients } \\
\hline Global LV-RS & 0.464 & 0.322 & 0.036 \\
\hline Global LV-CS & -0.422 & $-0.576^{*}$ & 0.554 \\
\hline Global LV-LS & -0.470 & $-0.591^{*}$ & 0.517 \\
\hline Global LV-3DS & 0.466 & 0.338 & 0.036 \\
\hline Global LV-AS & -0.460 & $-0.601^{*}$ & 0.567 \\
\hline \multicolumn{4}{|l|}{ In pcTOF patients } \\
\hline Global LV-RS & $0.535^{\star}$ & 0.371 & -0.480 \\
\hline Global LV-CS & -0.320 & -0.287 & $0.596^{*}$ \\
\hline Global LV-LS & -0.149 & -0.009 & 0.328 \\
\hline Global LV-3DS & 0.496 & 0.342 & -0.462 \\
\hline Global LV-AS & -0.229 & -0.141 & 0.492 \\
\hline
\end{tabular}

*, P<0.05. cTOF, corrected tetralogy of Fallot; etrTOF, early total reconstruction for patients with TOF; pcTOF, early palliation, late correction for patients with TOF; LV, left ventricular; RS, radial strain; CS, circumferential strain; LS, longitudinal strain; 3DS, threedimensional strain; AS, area strain.

capability of simultaneous assessment of all $\mathrm{LV}$ strain values at the same time from the same 3D dataset in complex CHDs. Interestingly, patients who underwent early total correction for TOF (etrTOF) have mostly preserved global, mean segmental, and regional strains, with mean segmental LV-RS and LV-3DS being supernormal in certain cases. These supernormal values might be part of a preclinical compensatory mechanism before manifest deterioration of LV function, but to confirm these suspicions, longitudinal studies are warranted. Another important finding is that for cTOF patients with early palliation-late correction (pcTOF), all global and mean segmental strain values were mostly significantly reduced compared to both controls and etrTOF subjects. For segmental strains, these differences were similarly present. A notable finding in segmental strain analysis is that mostly septal segments had significantly lower LV strains in pcTOF patients. This finding is most likely explained by the nature of TOF, the longer presence of the VSD, and the interventricular septal patch. Correlations could also be presented in cTOF patients between LV strains and aortic stiffness parameters, and those correlations were present during subgroup analysis, as well. These results confirm strong effects of ventriculararterial coupling in these patients in spite of the above presented abnormalities.

\section{Limitation section}

There are some limitations the authors consider important to take into account when interpreting results:

* Currently 3DSTE has lower temporal and spatial resolution than $2 \mathrm{D}$ echocardiography, therefore image quality is worse;

* It was not aimed to validate 3DSTE parameters due 
to their validated nature. Moreover, detailed analysis of other heart chambers was also not purposed to be performed;

* Interventricular and atrio-ventricular interactions are of high importance, the current study did not aim to evaluate them;

* A relatively small number of patients was investigated in a single adult CHD center. For better statistical strength, more patients could have been evaluated.

\section{Conclusions}

Significant LV deformation abnormalities could be demonstrated in cTOF patients. etrTOF patients have beneficial LV strain parameters as compared to those of pcTOF patients. LV strains show correlations with echocardiography-derived aortic elastic properties in cTOF patients suggesting ventricular-arterial coupling.

\section{Acknowledgments}

Funding: None.

\section{Footnote}

Reporting Checklist: The authors have completed the STROBE reporting checklist. Available at http://dx.doi. org/10.21037/cdt-20-366

Data Sharing Statement: Available at http://dx.doi. org/10.21037/cdt-20-366

Provenance and Peer Review: This article was commissioned by the Guest Editors (Yskert von Kodolitsch, Harald Kaemmerer, Koichiro Niwa) for the series "Current Management Aspects in Adult Congenital Heart Disease (ACHD): Part III" published in Cardiovascular Diagnosis and Therapy. The article has undergone external peer review.

Conflicts of Interest: All authors have completed the ICMJE uniform disclosure form (available at http:// dx.doi.org/10.21037/cdt-20-366). The series "Current Management Aspects in Adult Congenital Heart Disease (ACHD): Part III" was commissioned by the editorial office without any funding or sponsorship. The authors have no other conflicts of interest to declare.
Ethical Statement: The authors are accountable for all aspects of the work in ensuring that questions related to the accuracy or integrity of any part of the work are appropriately investigated and resolved. All cTOF patients and control subjects gave informed consent. The institutional human research committee approved the study which complied with the Declaration of Helsinki (as revised in 2013) (registration number: 71/2011).

Open Access Statement: This is an Open Access article distributed in accordance with the Creative Commons Attribution-NonCommercial-NoDerivs 4.0 International License (CC BY-NC-ND 4.0), which permits the noncommercial replication and distribution of the article with the strict proviso that no changes or edits are made and the original work is properly cited (including links to both the formal publication through the relevant DOI and the license). See: https://creativecommons.org/licenses/by-nc-nd/4.0/.

\section{References}

1. Francois K. Aortopathy associated with congenital heart disease: A current literature review. Ann Pediatr Cardiol 2015;8:25-36.

2. Swamy P, Bharadwaj A, Varadarajan P, et al. Echocardiographic evaluation of tetralogy of Fallot. Echocardiography 2015;32 Suppl 2:S148-S156.

3. Cruz C, Pinho T, Ribeiro V, et al. Aortic dilatation after tetralogy of Fallot repair: A ghost from the past or a problem in the future?. Rev Port Cardiol 2018;37:549-57.

4. Tan JL, Davlouros PA, McCarthy KP, et al. Intrinsic histological abnormalities of aortic root and ascending aorta in tetralogy of Fallot: evidence of causative mechanism for aortic dilatation and aortopathy. Circulation 2005;112:961-8.

5. Mohammad Nijres B, Bokowski J, Al-Kubaisi M, et al. Use of Speckle Tracking Echocardiography to Assess Left Ventricular Systolic Function in Patients with Surgically Repaired Tetralogy of Fallot: Global and Segmental Assessment. Pediatr Cardiol 2018;39:1669-75.

6. Niwa K. Aortopathy in Congenital Heart Disease in Adults: Aortic Dilatation with Decreased Aortic Elasticity that Impacts Negatively on Left Ventricular Function. Korean Circ J 2013;43:215-20.

7. Al Habib HF, Jacobs JP, Mavroudis C, et al. Contemporary patterns of management of tetralogy of Fallot: data from 
the Society of Thoracic Surgeons Database. Ann Thorac Surg 2010;90:813-9; discussion 819-20.

8. Havasi K, Kalapos A, Berek K, et al. More Than 50 Years' Experience in the Treatment of Patients With Congenital Heart Disease at a Hungarian University Hospital. Orv Hetil 2015;156:794-800.

9. Lang RM, Badano LP, Mor-Avi V, et al. Recommendations for cardiac chamber quantification by echocardiography in adults: an update from the American Society of Echocardiography and the European Association of Cardiovascular Imaging. Eur Heart J Cardiovasc Imaging 2015;16:233-70.

10. Nemes A, Geleijnse ML, Forster T, et al.

Echocardiographic evaluation and clinical implications of aortic stiffness and coronary flow reserve and their relation. Clin Cardiol 2008;31:304-9.

11. Nemes A, Kalapos A, Domsik P, et al. Three-dimensional speckle-tracking echocardiography -- a further step in non-invasive three-dimensional cardiac imaging. Orv Hetil 2012;153:1570-7.

12. Zhang J, Chowienczyk PJ, Spector TD, et al. Relation of arterial stiffness to left ventricular structure and function

Cite this article as: Rácz G, Kormányos Á, Domsik P, Kalapos A, Gyenes N, Havasi K, Ambrus N, Hartyánszky I, Bogáts G, Nemes A. Left ventricular strains correlate with aortic elastic properties in adult patients with corrected tetralogy of Fallot (Results from the CSONGRAD Registry and MAGYARPath Study). Cardiovasc Diagn Ther 2021;11(2):611-622. doi: 10.21037/cdt-20-366 in healthy women. Cardiovasc Ultrasound 2018;16:21.

13. Nemes A, Kalapos A, Domsik P, et al. Correlations between echocardiographic aortic elastic properties and left ventricular rotation and twist--insights from the three-dimensional speckle-tracking echocardiographic MAGYAR-Healthy Study. Clin Physiol Funct Imaging 2013;33:381-5.

14. van Grootel RWJ, van den Bosch AE, Baggen VJM, et al. The Prognostic Value of Myocardial Deformation in Adult Patients With Corrected Tetralogy of Fallot. J Am Soc Echocardiogr 2019;32:866-875.e2.

15. Cruz C, Pinho T, Sousa C, et al. Ascending aorta in tetralogy of Fallot: Beyond echocardiographic dimensions. Echocardiography 2018;35:1362-9.

16. Menting ME, Eindhoven JA, van den Bosch AE, et al. Abnormal left ventricular rotation and twist in adult patients with corrected tetralogy of Fallot. Eur Heart J Cardiovasc Imaging 2014;15:566-74.

17. Li SN, Yu W, Lai CT, et al. Left ventricular mechanics in repaired tetralogy of Fallot with and without pulmonary valve replacement: analysis by three-dimensional speckle tracking echocardiography. PLoS One 2013;8:e78826. 